\title{
HLA Antigens in Japanese Patients with Myasthenia Gravis
}

\author{
Kazamasa Matsuki," Takeo Juji," Katsushi Tokunaga," Mamoru Takamizawa," Hiroo Maeda," Mariko Soda," \\ Yoshiko Nomura," and Masaya Segawal" \\ *Blood Transfusion Service, Tokyo University Hospital, Hongo, Bunkyo-ku, Tokyo 113, ${ }^{\ddagger}$ Department of Anthropology, Faculty of \\ Science, University of Tokyo, Hongo, Bunkyo-ku, Tokyo 113, ${ }^{8}$ Blood Transfusion Service, Saitama Medical School, Kamoda, \\ Kawagoe, Saitama 350, and "Segawa Neurological Clinic for Children, Kanda, Chiyoda-ku, Tokyo 101, Japan
}

\begin{abstract}
HLA antigens in 104 Japanese patients and 41 families with myasthenia gravis (MG) were investigated. The frequencies of DR9 and DRw13 were significantly increased in the patients who developed MG before 3 yr of age. The DQw3 antigen was positive for all the patients that developed MG before 15 yr with only one exception. All the examined cases that developed MG before 3 yr (including this DQw3 negative patient) had the same DQA and DQB DNA restriction fragments. These HLA frequencies decreased as the age of onset increased, and no significant association was observed in adult-onset MG. No patients had B8, DR3, and DQw2.

The relative risk was higher for the DR9/DRw13 heterozygotes (37.4) than for DR9 (16.4) or DRw13 (7.1) in the childhood-onset MG. Statistical analysis suggested that DR9. and DRw13 (or DQw1 and DQw3) act synergistically in the disease development.

Family study revealed diverse DR9 haplotypes. The most frequent DRw13 haplotype was Bw44-BFF-C4A3B1-DRw13DQw1, which may be evolutionarily related to the caucasian B8-DR3-DQw2 haplotype. These results showed that MG in early childhood in Japanese individuals is genetically different from that in adulthood and that in caucasians. (J. Clin. Invest. 1990. 86:392-399.) Key words: myasthenia gravis • HLA antigens $\bullet$ disease association - restriction fragment length polymorphison
\end{abstract}

\section{Introduction}

Myasthenia gravis (MG) ${ }^{1}$ is an autoimmune disorder that affects neuromuscular junctions (1). The clinical symptoms of myasthenia gravis include muscle weakness after repeated exercise, diurnal fluctuation of the symptoms, frequent concurrence and family history of other immunological disorders such as asthma, thyroiditis, and atopic dermatitis. Muscle weakness is markedly improved by the Tensilon test. Laboratory examinations generally reveal frequent occurrence of thymus enlargement or thymoma, presence of antiacetylcholine receptor antibodies, and waning phenomenon on electromyographic examination (1).

Address reprint requests to Dr. Matsuki, Immunobiology Research Center, Box 724, 420 Delaware St., S. E., Minneapolis, MN 55455.

Received for publication 18 January 1990 and in revised form 4 April 1990

1. Abbreviations used in this paper: MG, myasthenia gravis; RFLP, restriction fragment length polymorphism.

J. Clin. Invest.

(C) The American Society for Clinical Investigation, Inc.

0021-9738/90/08/0392/08 $\$ 2.00$

Volume 86, August 1990, 392-399
One of the important features in this disorder is its strong association with the HLA antigens (2-4). In caucasians, a number of studies showed firm association between this disorder and HLA-A1, B8, and DR3 (3-5). In Orientals, however, the association has been ambiguous. The 8th International Histocompatibility Workshop (6), for example, failed to find any significant association between the disorder and HLA in Orientals.

To date, most of the studies on HLA in Oriental MG have been limited to the patients who developed the disorder in the adulthood $(7,8)$. However, in Japanese the large number of the patients develop the disorder before 3 yr of age $(9,10)$. The age at onset of MG in Japanese shows a bimodal distribution: the first and highest peak is before the age of $3 \mathrm{yr}$ and the second and lower peak in the third decade $(9,10)$. This is in contrast to caucasian MG, in which the distribution generally shows a single adulthood peak (1): in the third decade for females and in the fifth or sixth decades for males. In this study, we investigated the HLA antigens in a total of 104 Japanese MG patients that developed the disorder at various ages, from 1 mo to $67 \mathrm{yr}$. We also analyzed HLA haplotypes in 41 families.

\section{Methods}

Patients. Patients were 104 randomly selected Japanese $(24$ males and 80 females) outpatients at Segawa Neurological Clinic for Children (Chiyoda-ku, Tokyo) and the Department of Neurology at the Juntendo University (Bunkyo-ku, Tokyo) (Table I). The diagnosis was made clinically before HLA typing. All the patients met the standard diagnostic criteria for MG that were reevaluated by the Japanese Committee on Neuroimmunological Disorders (11). All of them showed muscle weakness after repeated exercise, diurnal fluctuation of the symptoms, and marked effectiveness of the Tensilon test. The clinical details and diagnosis of the patients who developed the disorder in the childhood were described elsewhere (12-14). All the patients reported in the previous studies were included (12-16). Patients with neonatal and congenital MG or those having thymoma were excluded from the present study. The patients were arbitrarily classified into four subgroups according to the age at onset $(13,14)$ : subgroup I includes 43 patients that developed the disorder before the age of $3 \mathrm{yr}$; subgroup II includes 20 patients that developed MG between 3 and $5 \mathrm{yr}$ of age; subgroup III includes 18 patients between 6 and 14; and subgroup IV includes 23 patients after $15 \mathrm{yr}$ of age (Table I). Controls were 220 apparently healthy randomly selected Japanese.

Family study. A total of 41 families were selected and each family member was examined to deduce the HLA haplotypes. Four families were intentionally included since two of the families had monozygotic twins, one had dizygotic twins, and the other had two children with MG; however, all the other families were selected at random. Among the 41 families, 21 of the probands (families 1-21) belonged to the subgroup I, 10 (families 22-31) to the subgroup II, and 10 (families $32-41$ ) to the subgroup III. In this study, no family with a proband in subgroup IV was included. The haplotype and segregation data of the control Japanese families were obtained from the 8th Japan HLA workshop data (17). 
Table I. Patient Subgroups

\begin{tabular}{ccccc}
\hline Subgroup* & Age at onset & Male & Female & Total \\
\hline & $y r$ & $n$ & $n$ & $n$ \\
I & $0-2$ & 10 & 33 & 43 \\
II & $3-5$ & 6 & 14 & 20 \\
III & $6-14$ & 5 & 13 & 18 \\
IV & $15-$ & 3 & 20 & 23 \\
Total & & 24 & 80 & 104 \\
\hline
\end{tabular}

* Subgroups were according to Segawa (13) and Segawa et al. (14).

HLA typing. Serological HLA typing was performed by the modified two-stage complement-dependent microcytotoxicity method as described previously (18). The typing sera were obtained at Blood Transfusion Service, Tokyo University Hospital, or through serum exchange. The typing sera distributed at the 9 th and 10th International Histocompatibility Workshop were also used to determine HLA antigens. At least three sera were used to type a single HLA specificity. Complement allotypes were typed according to the method of Sim and Cross (19) and to the method of Alper and others (20).

Statistical analysis. For comparing HLA antigen frequencies between the patients and healthy subjects, $P$ values were obtained by the chi square or the Fisher exact test. Corrected $P$ values $(P c)$ were calculated as $P c=1-(1-P)^{n}$, where $n$ stands for the number of the antigens examined or the number of detected polymorphic restriction fragments. Relative risks were calculated by the method of Woolf (21). To analyze synergistic interaction statistically, the $S$ values were calculated according to the method of Rothman (22).

Two different kinds of genetic models were examined to see if they could explain the observed HLA phenotype frequencies. The first model assumes a single disease-predisposing gene that is in linkage disequilibrium with only one HLA antigen such as DR9. The second model assumes a single disease gene that is in linkage disequilibrium with two HLA antigens such as DR9 and DRw13. Both dominant and recessive modes of inheritance were evaluated for each model. For the first model, expected genotype frequencies under the dominant inheritance were calculated by the moment method as described by Thomson (23) and those under the recessive inheritance were calculated by the counting method of maximum likelihood as described previously (18). For the second model, the expected frequencies were computed according to the maximum likelihood method (23). Attributable risks were calculated according to Thomson (24).

Southern analysis. Among the HLA-typed patients in the subgroup I (age at onset $<3 \mathrm{yr}$ ), patients were further studied for the DQA, DQB, and DPB restriction fragment length polymorphisms (RFLP). One DQw3-negative patient was intentionally selected, while the other DQw3-positive patients were randomly selected from the remaining patients. Southern analysis was performed as described previously (25). Briefly, genomic DNA was isolated from the peripheral blood leukocytes (23). The DNA samples were digested by Taq I, Eco RI, Pst $\mathrm{I}$, and Msp I, and the digested DNA (8-12 $\mu \mathrm{g})$ was electrophoresed on a $0.65 \%$ agarose gel and transferred to the nylon membrane. The filters were prehybridized overnight at $65^{\circ} \mathrm{C}$ in bags containing $5 \times$ Denhardt's solution $(0.1 \%$ bovine serum albumin, $0.1 \%$ Ficoll type 400 , $0.1 \%$ polyvinylpyrrolidone), $5 \times \operatorname{SSPE}(0.9 \mathrm{M} \mathrm{NaCl}, 5 \mathrm{mM}$ EDTA, 50 $\mathrm{mM}$ sodium phosphate buffer, $\mathrm{pH} 7.4), 0.5 \% \mathrm{SDS}$, and $200 \mu \mathrm{g} / \mathrm{ml}$ sheared, denatured salmon sperm DNA. They were then hybridized with ${ }^{32}$ P-labeled cDNA DQA, DQB, and DPB probes (25) (labeled by the random primer method to the specific activity over $1 \times 10^{8}$ $\mathrm{cpm} / \mu \mathrm{g}$ ) for $40 \mathrm{~h}$ at $65^{\circ} \mathrm{C}$ in the same buffer as that used for prehybridization. Filters were washed twice at room temperature for $5 \mathrm{~min}$ in $2 \times$ SSC ( $1 \times$ SSC: $150 \mathrm{mM} \mathrm{NaCl}, 15 \mathrm{mM}$ sodium citrate); once at $65^{\circ} \mathrm{C}$ for $30 \mathrm{~min}$ in $2 \times \mathrm{SSC}$; once at $65^{\circ} \mathrm{C}$ for $30 \mathrm{~min}$ in $2 \times \mathrm{SSC}, 0.5 \%$
SDS; and once at $65^{\circ} \mathrm{C}$ for $30 \mathrm{~min}$ in $0.1 \times \mathrm{SSC}$. Filters were exposed to Kodak XAR-5 films for $5-10 \mathrm{~d}$ at $-70^{\circ} \mathrm{C}$ using Dupont Lightning Plus intensifying screens.

\section{Results}

HLA antigen frequencies. Table II shows the HLA antigen frequencies in Japanese patients with myasthenia gravis subclassified according to the age at onset. As for class I antigens, only the frequencies of Aw33 and B44 were increased significantly in the subgroup I as compared with the healthy control. No patients had B8, which is associated with MG in caucasians (2-5).

As for class II antigens, the frequencies of DR9 and DRw13 were both increased significantly in the subgroup I as compared with the control. The relative risks for DR9 and DRw13 in subgroup I were 16.4 and 7.1, respectively. Fig. 1 plots the DR antigen frequencies in each case and surrounding 12 cases combined against the age of onset of the case. These frequencies decreased rapidly until $10 \mathrm{yr}$ of age and reached the almost constant level, although the DR9 frequency displayed a small increase in the third decade (Fig. 1).

The DRw13 frequency was slightly higher in males (8 in $10,80 \%)$ than in females $(17$ in $33,52 \%)$ in subgroup I, but this difference was not statistically significant. No antigens showed significant difference between sexes in each subgroup.

The HLA-DR antigen frequencies in subgroup I were significantly different from those in all the other subgroups combined $\left(\chi_{\text {d.f. }=4}^{2}=24.7, P<0.0001\right)$.

As for the DQ antigens, all but one patient in the subgroups I through III were DQw3 positive (relative risk of 28.0 in subgroup I). The DQw3 frequency remained almost $100 \%$ before $15 \mathrm{yr}$ of age at onset; after $15 \mathrm{yr}$, it declined (Fig. 2). The DQw 1 frequency showed a bimodal distribution: the first peak before the age of 5 , and the second peak in the third decade. The

Table II. HLA Antigen Frequencies in Myasthenia Gravis

\begin{tabular}{lccccc}
\hline & & \multicolumn{4}{c}{ Age at onset (in years) } \\
\cline { 3 - 6 } Antigen & $\begin{array}{c}\text { Control } \\
(n=220)\end{array}$ & $\begin{array}{c}0-2 \\
(n=43)\end{array}$ & $\begin{array}{c}3-5 \\
(n=20)\end{array}$ & $\begin{array}{c}6-15 \\
(n=18)\end{array}$ & $\begin{array}{c}16- \\
(n=23)\end{array}$ \\
\hline Aw33 & $36(16 \%)$ & $22(51 \%)^{*}$ & $6(30 \%)$ & $3(17 \%)$ & $3(13 \%)$ \\
B8 & $2(1 \%)$ & $0(0 \%)$ & $0(0 \%)$ & $0(0 \%)$ & $0(0 \%)$ \\
B44 & $28(13 \%)$ & $22(51 \%)^{*}$ & $7(35 \%)$ & $2(11 \%)$ & $2(9 \%)$ \\
DR1 & $14(6 \%)$ & $2(5 \%)$ & $2(10 \%)$ & $0(0 \%)$ & $1(4 \%)$ \\
DR2 & $66(30 \%)$ & $3(7 \%)$ & $1(5 \%)$ & $3(17 \%)$ & $9(39 \%)$ \\
DR3 & $2(1 \%)$ & $0(0 \%)$ & $0(0 \%)$ & $0(0 \%)$ & $0(0 \%)$ \\
DR4 & $84(38 \%)$ & $5(12 \%)^{\ddagger}$ & $4(20 \%)$ & $5(28 \%)$ & $7(30 \%)$ \\
DRw8 & $60(27 \%)$ & $8(19 \%)$ & $9(45 \%)$ & $6(33 \%)$ & $7(30 \%)$ \\
DR9 & $60(27 \%)$ & $37(86 \%)^{*}$ & $13(65 \%)^{\ddagger}$ & $9(50 \%)$ & $10(43 \%)$ \\
DRw11 & $7(3 \%)$ & $0(0 \%)$ & $1(5 \%)$ & $2(11 \%)$ & $0(0 \%)$ \\
DRw12 & $29(13 \%)$ & $4(9 \%)$ & $2(10 \%)$ & $5(28 \%)$ & $3(13 \%)$ \\
DRw13 & $36(16 \%)$ & $25(58 \%)^{*}$ & $7(35 \%)$ & $5(28 \%)$ & $2(9 \%)$ \\
DRw14 & $22(10 \%)$ & $0(0 \%)$ & $0(0 \%)$ & $0(0 \%)$ & $4(17 \%)$ \\
DRw52 & $126(57 \%)$ & $35(81 \%)$ & $17(85 \%)$ & $17(94 \%)$ & $13(57 \%)$ \\
DRw53 & $134(61 \%)$ & $40(93 \%)^{4}$ & $17(85 \%)$ & $13(72 \%)$ & $15(65 \%)$ \\
DQw1 & $143(65 \%)$ & $35(81 \%)$ & $14(70 \%)$ & $8(44 \%)$ & $18(78 \%)$ \\
DQw2 & $2(1 \%)$ & $0(0 \%)$ & $0(0 \%)$ & $0(0 \%)$ & $0(0 \%)$ \\
DQw3 & $132(60 \%)$ & $42(98 \%)^{\prime \prime}$ & $20(100 \%)^{*}$ & $18(100 \%)$ & $16(70 \%)$ \\
DQw4 & $62(28 \%)$ & $2(5 \%)$ & $1(5 \%)$ & $6(33 \%)$ & $4(17 \%)$ \\
DQw7 & $43(20 \%)$ & $4(9 \%)$ & $3(15 \%)$ & $7(39 \%)$ & $4(17 \%)$ \\
& & & & & \\
& & & & &
\end{tabular}

* $P c<0.0001$; ${ }^{\star}$ Significant increase or decrease as compared with controls at $P \mathrm{c}<0.05 ;{ }^{8} \mathrm{Pc}<0.01 ;{ }^{\|} \mathrm{Pc}<0.001$. 


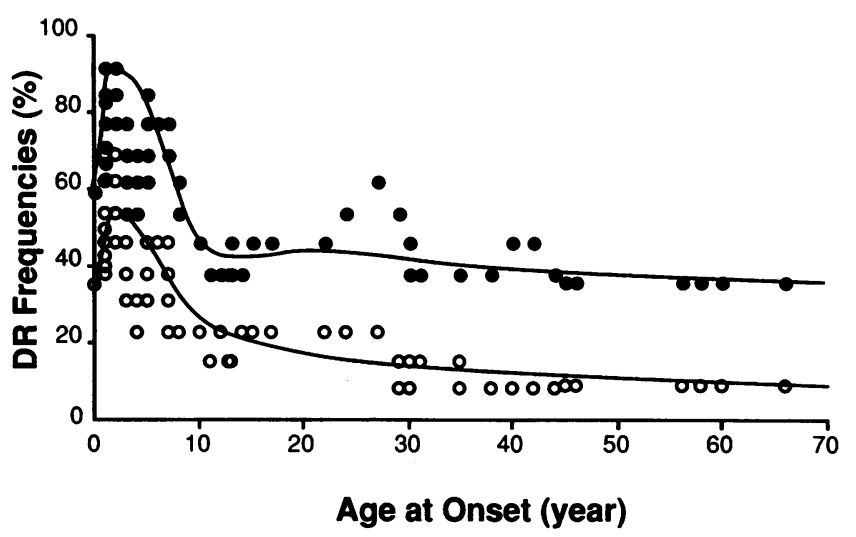

Figure 1. DR9 and DRw13 antigen frequencies and the age at onset. Each dot represents a frequency of a DR antigen in the case and surrounding 12 cases with close age of onset. (Closed circles) DR9 frequencies; (open circles) DRw13 frequencies.

DQw4 and DQw7 showed patterns complimentary to that of DQw1, with a peak of $\sim 10$ yr of age. No patients had DR3 or DQw2, which are frequently found in caucasian patients with MG.

Genotype frequencies. In the subgroup I, the number of DR9/DRw13 heterozygotes was increased significantly as compared with that in controls $\left(\chi^{2}=88.7, P<10^{-20}\right)$ (Table III). The relative risk to develop MG was 37.4 for the DR9/ DRw 13 heterozygotes. Although there was one DR9 homozygote in the subgroup I, there was no DRw 13 homozygote in all the patient groups. The relative risks for DR9/DRw13 were not as high in other subgroups as in subgroup I. The DQw1/ DQw3 heterozygotes were also significantly increased in the subgroup I (Table III).

Synergistic interaction. The $S$ value for DR9 and DRw 13 computed by the method of Rothman (22) was 8.63 in subgroup I. This indicates that the observed risk for the DR9/ DRw1 3 subjects to develop MG was 8.63 times higher than the expected value under the assumption that DR9 and DRw13 has no synergistic interaction $(P<0.0001)$. However, in subgroups II, III, and IV, no statistically significant synergistic interaction was observed.

Single disease allele model. To investigate the underlying genetics in early childhood-onset MG, two sets of genetic

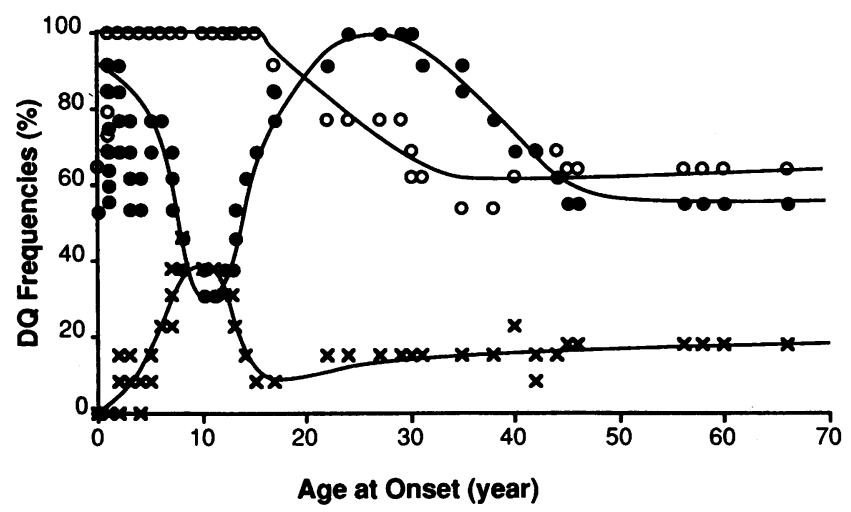

Figure 2. DQ antigen frequencies and the age at onset. (Closed circles) DQw1; (open circles) DQw3; (crosses) DQw4.
Table III. DR and DQ Genotype Frequencies

\begin{tabular}{|c|c|c|c|c|c|c|}
\hline \multirow[b]{2}{*}{ Groups } & \multicolumn{6}{|c|}{ N of DR Genotypes (relative risk) } \\
\hline & 9/-* & $9 / w 13$ & $w 13 /-$ & $9 / x^{\ddagger}$ & $w 13 / x$ & $\mathbf{x} / \mathbf{x}$ \\
\hline $\begin{array}{l}\text { Controls } \\
\text { Patients }\end{array}$ & 5 & 6 & 2 & 25 & 14 & 168 \\
\hline I & $1(1.0)$ & $22(37.4)^{\S}$ & $0(0)$ & $14(3.8)^{\prime \prime}$ & $3(1.1)$ & $3(0)^{1}$ \\
\hline II & $0(0)$ & $4(8.9)$ & $0(0)$ & $9(6.4)$ & $3(2.6)$ & $4(0.1)^{11}$ \\
\hline III & $0(0)$ & $5(13.7)^{* *}$ & $0(0)$ & $4(2.2)$ & $0(0)$ & $9(0.3)$ \\
\hline IV & $0(0)$ & $1(1.6)$ & $0(0)$ & $9(5.0)$ & $1(0.7)$ & $12(0.3)$ \\
\hline
\end{tabular}

b. DQ genotypes

\begin{tabular}{clccccc}
\hline & \multicolumn{6}{c}{ N of DQ Genotypes (relative risk) } \\
\cline { 2 - 7 } Groups & w1/ & w1/w3 & w3/ & w1/w4 & w3/w4 & w4/- \\
\hline Controls & 37 & 105 & 29 & 20 & 18 & 11 \\
Patients & & & & & & \\
I & $1(0.1)$ & $34(4.1)^{\prime \prime}$ & $6(1.1)$ & $0(0)$ & $2(0.6)$ & $0(0)$ \\
II & $0(0)$ & $14(2.6)$ & $5(2.2)$ & $0(0)$ & $1(0.6)$ & $0(0)$ \\
III & $0(0)$ & $8(0.9)$ & $4(1.9)$ & $0(0)$ & $6(5.6)$ & $0(0)$ \\
IV & $5(1.4)$ & $11(1.0)$ & $3(1.0)$ & $2(1.0)$ & $2(1.1)$ & $0(0)$
\end{tabular}

* Homozygotes or possible heterozygotes having DR "blank" antigens; ${ }^{\ddagger} \mathrm{x}, \mathrm{DR}$ antigens other than DR9 and DRw $13 ;{ }^{8} P<0.00001$; " $P<0.0001$; $P<0.000001$; ** Significant increase or decrease as compared with controls by the chi-square test or the Fisher exact test at $P<0.001$.

models were evaluated in subgroup I. Under the assumption of a single disease-predisposing gene in linkage disequilibrium to a single HLA-DR9 antigen, the excepted numbers of the DR9 homozygotes and heterozygotes were calculated as 5.33 and 31.67 in the dominant model and 16.88 and 20.12 in the recessive model. These numbers do not fit the observed numbers of 1 and $36\left(P<0.05\right.$ for the dominant and $P<10^{-6}$ for the recessive models). Similarly, single disease allele models in linkage with only DRw13 failed to explain the DRw13 genotype frequencies $(P<0.05$ for the dominant and $P<0.001$ for the recessive models).

Another model is the one that assumes a single diseasepredisposing allele that is in linkage disequilibrium with both DR9 and DRw13. Table IV shows the expected DR9 and DRw13 genotype frequencies calculated under strict dominant and recessive models of the disease allele. Both models were rejected (the recessive model at $P<0.02$, the dominant model at $P<0.003$.) Similar analysis also rejected a single disease allele model in linkage disequilibrium with both DQw 1 and DQw3 (Table IV). However, this discrepancy between the observed HLA frequencies and the expected frequencies under this model meshed well with the three-allele synergistic model proposed by Louis and Thomson (26). According to this model, DR9 (or DQw3) or DRw13 (or DQw1) acts as a dominant or a recessive gene in the absence of the other, respectively.

Family study. To analyze disease associated haplotypes, we have studied 41 families with the probands who developed 
Table IV. Genetic Models in Subgroup I under the Assumption that a Single Disease-predisposing Gene Is Linked with Two HLA Alleles

\begin{tabular}{|c|c|c|c|c|c|c|}
\hline DR Genotypes & 9/-* & $9 / w 13$ & w13/- & $9 / x^{\ddagger}$ & w13/x & $\mathbf{x} / \mathbf{x}$ \\
\hline Observed N & 1 & 22 & 0 & 14 & 3 & 3 \\
\hline \multicolumn{7}{|l|}{ Expected $\mathrm{N}$} \\
\hline Dominant & 3.95 & 4.21 & 1.11 & 20.91 & 10.20 & 2.62 \\
\hline $\mathrm{k} 1=0.60 ; \mathrm{k} 2=0.31^{\S}$ & \multicolumn{4}{|c|}{$\chi_{\text {d.f. }=4}^{2}=16.14$} & \multicolumn{2}{|l|}{$P=0.0028$} \\
\hline Recessive & 8.40 & 11.05 & 3.63 & 10.16 & 6.69 & 3.08 \\
\hline $\mathrm{k} 1=0.44 ; \mathrm{k} 2=0.29$ & \multicolumn{4}{|c|}{$\chi_{\mathrm{d} . \mathrm{f} .=4}^{2}=12.88$} & \multicolumn{2}{|l|}{$P=0.012$} \\
\hline DQ Genotypes & w1/- & $\mathrm{w} 1 / \mathrm{w} 3$ & w3/- & $w 1 / w 4$ & $w 3 / w 4$ & $w 4 /-$ \\
\hline Observed N1 & 1 & 34 & 6 & 0 & 2 & 0 \\
\hline \multicolumn{7}{|l|}{ Expected N } \\
\hline \multicolumn{7}{|l|}{ Dominant } \\
\hline$k 1=0.03 ; k 2>1$ & \multicolumn{6}{|c|}{ (This model was rejected because $k 2$ was greater than 1. ) } \\
\hline Recessive & 9.45 & 25.88 & 17.72 & 1.23 & 1.69 & 0.04 \\
\hline $\mathrm{k} 1=0.42 ; \mathrm{k} 2=0.56$ & \multicolumn{3}{|c|}{$\chi_{\text {d.f. }=2}^{2}=11.12$} & \multicolumn{3}{|c|}{$P=0.003$} \\
\hline
\end{tabular}

*-Homozygotes or possible heterozygotes having DR "blank" antigens; ${ }^{*} \mathrm{x}, \mathrm{DR}$ antigens other than DR9 and DRw13. ${ }^{8} \mathrm{The} k 1$ and $\mathrm{k} 2 \mathrm{values}$ and the expected number of each phenotype were obtained according to Thomson (23). The $\mathrm{k} 1$ and $\mathrm{k} 2$ values represent the proportions of the DR9 and DRw13 (or DQw1 and DQw3) haplotypes in the disease susceptibility haplotypes. To calculate chi square values, categories with small number were added to make each category bigger than 5 . The patients analyzed here were the probands of subgroup I. The control DR9 and DRw1 3 allele frequencies were calculated from the controls here.

MG before $15 \mathrm{yr}$ of age (Fig. 3). In the families with two monozygotic twins (families 11 and 22) and one dizygotic twin (family 6), only one of each twin developed MG. In the family with two affected children (family 7), all the HLA haplotypes were different between the children.

In these 41 families, 31 probands had a DR9 haplotype (Table V). Among these DR9 haplotypes, the most frequent haplotype was Bw61-DR9, which is also the most frequent haplotype in the normal Japanese population. The second most frequent haplotype in the patients was Cw11-Bw46-DR9. The complotype BFS-C4A4-C4B2 was linked with this haplotype in all of the cases. This haplotype is quite rare in control Japanese (17). This complotype was the same as that found in the Chinese Cw11-Bw46-DR9 haplotype.
Among 18 DRw13 haplotypes, 16 haplotypes (89\%) were C blank-B44-DRw13-DQw1. This haplotype was found in 42 $(61 \%)$ of the 69 DRw13 haplotypes in healthy Japanese families $(P=0.02$ by Fisher exact test). All the 16 haplotypes carried the complotype BFF-C4A3-C4B1.

$H L A-D Q$ and DPB RFLP. A RFLP analysis using the DQB probe showed an increased frequency of the Eco RI 20 -kb fragment $(P<0.00001)$ and a $100 \%$ presence of the Taq I 1.9-kb fragment $(P<0.003)$ (including one $D Q w 3$ negative case) (Table VI). Representative blots are shown in Fig. 4. The DQA1 RFLP also revealed complete presence of the Taq I 5.4-kb fragment $(P<0.03)$. As for the DQA2 and DPB RFLP, none of the polymorphic fragments showed significant deviation.

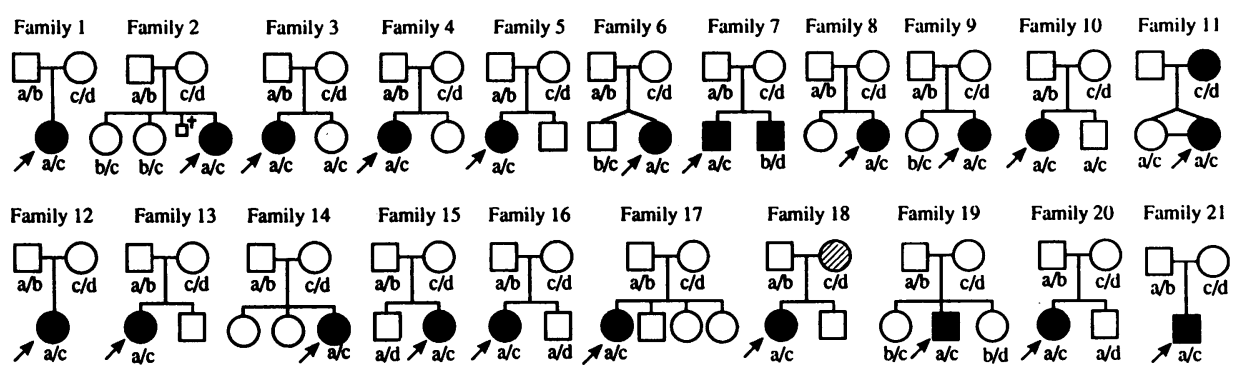

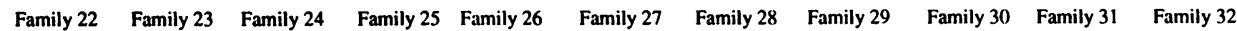
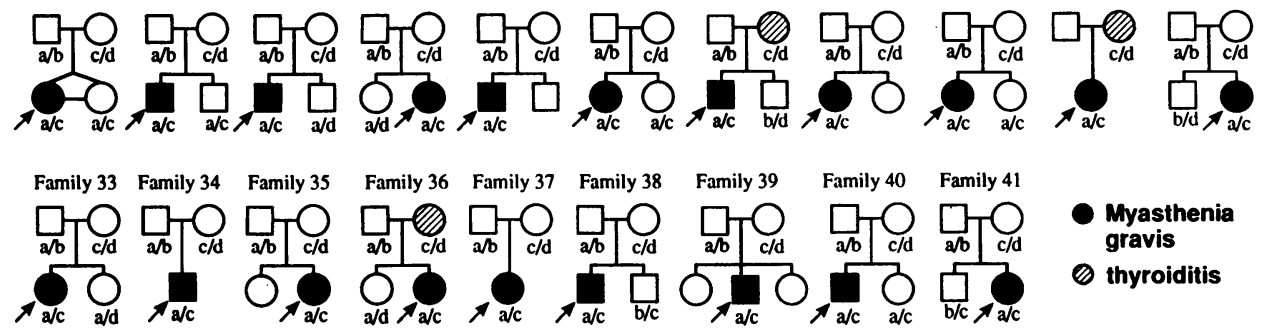

Figure 3. Family segregation study. (Arrow) proband; (closed circle) patients with MG; (hatched circle) subjects with thyroiditis; $a, b, c, d$, HLA haplotypes. 
Table V. Distribution of the DR9 Haplotypes in Healthy Japanese and the Patients with Childhood-onset MG

\begin{tabular}{llc}
\hline DR9 haplotype & MG $(n=31)$ & $\begin{array}{c}\text { Control } \\
(n=133)\end{array}$ \\
\hline Bw61-DR9 & $9(29.0 \%)$ & $30(22.5 \%)$ \\
B51-DR9 & $3(9.7 \%)$ & $20(15.0 \%)$ \\
Bw62-DR9 & $0(0.0 \%)$ & $13(9.9 \%)$ \\
Bw60-DR9 & $1(3.2 \%)$ & $12(9.0 \%)$ \\
B35-DR9 & $4(12.9 \%)$ & $9(6.8 \%)$ \\
B55-DR9 & $3(9.7 \%)$ & $7(5.3 \%)$ \\
B48-DR9 & $1(3.2 \%)$ & $5(3.8 \%)$ \\
Bw46-DR9 & $5(16.1 \%)^{*}$ & $4(3.0 \%)$ \\
Others & $5(16.1 \%)$ & $33(24.8 \%)$
\end{tabular}

* Significant increase at $P=0.02$ by the Fisher exact test.

We studied all the possible combination of the DQA, DQB genes and DR specificities in the 23 patients that were examined for both DQA and DQB polymorphisms (Table VII). They belong all to subgroup I. Among them, 18 (78\%) were DQA Taq I $6.0 \mathrm{~kb}+/ \mathrm{DQB}$ Taq I $1.9 \mathrm{~kb}+; 17$ (74\%) DQA Taq I $6.0 \mathrm{~kb}+/ \mathrm{DR} 9+; 12$ (52\%) DQA Taq I $5.4 \mathrm{~kb}+/ \mathrm{DRw} 13+$; and 14 (61\%) DQA Taq I $5.4 \mathrm{~kb}+/$ DQB Eco RI $2.4 \mathrm{~kb}+$ (Table VII).

\section{Discussion}

This is the first study that demonstrates strong association between the HLA antigens and MG in Japanese. This is also

Table VI. RFLP in Myasthenia Gravis (Subgroup I)

\begin{tabular}{|c|c|c|c|c|c|c|}
\hline $\begin{array}{l}\text { Probe \& } \\
\text { enzyme }\end{array}$ & $\begin{array}{l}\text { Fragment } \\
\text { size }^{*}\end{array}$ & Controls & MG & $P c^{\ddagger}$ & $\begin{array}{l}\text { Relative } \\
\text { risk }\end{array}$ & $\begin{array}{c}\text { Attributable } \\
\text { risk }\end{array}$ \\
\hline & $k b$ & & & & & \\
\hline DQA 1 & & $(n=34)$ & $(n=29)$ & & & \\
\hline \multirow[t]{5}{*}{ Taq I } & 6.6 & $12(35 \%)$ & $5(17 \%)$ & & & \\
\hline & 6.0 & $14(41 \%)$ & $22(76 \%)$ & & & \\
\hline & 5.4 & $19(56 \%)$ & $29(100 \%)$ & 0.03 & 46.9 & 1.00 \\
\hline & 4.6 & $6(18 \%)$ & $0(0 \%)$ & & & \\
\hline & 2.4 & $5(15 \%)$ & $2(7 \%)$ & & & \\
\hline \multicolumn{7}{|l|}{ DQA2 } \\
\hline \multirow[t]{2}{*}{ Taq I } & 2.1 & $14(41 \%)$ & $20(69 \%)$ & & & \\
\hline & 1.9 & $32(94 \%)$ & 27 (93\%) & & & \\
\hline DQB & & $(n=28)$ & $(n=23)$ & & & \\
\hline \multirow[t]{5}{*}{ Eco RI } & 29 & $18(64 \%)$ & $9(39 \%)$ & & & \\
\hline & 20 & $9(39 \%)$ & $22(96 \%)$ & 0.00001 & 46.4 & 0.94 \\
\hline & 18 & $8(35 \%)$ & $1(4 \%)$ & & & \\
\hline & 5.0 & $1(4 \%)$ & $1(4 \%)$ & & & \\
\hline & 2.3 & $11(39 \%)$ & $14(61 \%)$ & & & \\
\hline \multirow[t]{3}{*}{ Taq I } & 6.0 & $8(35 \%)$ & $13(57 \%)$ & & & \\
\hline & 3.0 & $7(25 \%)$ & $2(8 \%)$ & & & \\
\hline & 1.9 & $18(64 \%)$ & $23(100 \%)$ & 0.003 & 26.7 & 1.00 \\
\hline DPB & & $(n=40)$ & $(n=29)$ & & & \\
\hline \multirow[t]{3}{*}{ Msp I } & 3.8 & $33(83 \%)$ & $24(83 \%)$ & & & \\
\hline & 2.4 & $24(60 \%)$ & $16(55 \%)$ & & & \\
\hline & 0.8 & $32(80 \%)$ & $27(93 \%)$ & & & \\
\hline \multirow[t]{2}{*}{ Pst I } & 3.2 & $31(78 \%)$ & $25(86 \%)$ & & & \\
\hline & 2.6 & $28(70 \%)$ & $26(90 \%)$ & & & \\
\hline
\end{tabular}

* Only polymorphic bands are shown.

₹ Corrected $P$ values $(P c)$ were obtained as $P c=1-(1-P)^{n}$, where $n$ is the number of detected polymorphic bands. $P$ values were calculated by the chisquare analysis or the Fisher exact test. the first study that analyzed extensively the HLA antigens in Japanese children with MG.

The HLA specificities that showed strong association were DR9, DRw13, DQw1, DQw3, and related DQA1 and DQB1 gene restriction fragments. The increase of Aw33 and B44 would be secondary to the DRw13 increase, since Aw33 and B44 have a strong linkage disequilibrium with DRw13 in Japanese (17). No patients had DR3 or DQw2. Since DR3 and DQw2 are associated with caucasian MG (2-5), our results demonstrated that Japanese MG differs from caucasian MG in the immunogenetic respect.

The strength of association was dependent on the age of onset. The association was strongest before $3 \mathrm{yr}$ of age, then it decreased with the age of onset. Pirskanen et al. (4) and Fritze et al. (2) showed the HLA-B8 and MG association was stronger in caucasian patients with age at onset earlier than $35 \mathrm{yr}$ and $40 \mathrm{yr}$, respectively. Yoshida et al. (7) showed B12 was more frequently found in Japanese patients that developed MG earlier than $30 \mathrm{yr}$. However, none of these previous studies included cases with age at onset as early as ours.

The difference in the HLA frequencies in the age at onset is striking. To date, only few diseases have been known to show such great difference. One of the disorders is diabetes mellitus. In diabetes mellitus, there is a clear HLA association in the childhood-onset type, while no strong association in the adultonset type (5). This phenomenon is explained by the fact that although both diseases share similar clinical symptoms the underlying pathophysiologies are different. The other one is rheumatoid arthritis. Rheumatoid arthritis is associated with DR4 in the adult type and with B27 and DR5 in the juvenile type (5). It is postulated that juvenile rheumatoid arthritis comprises several different subtypes and the majority of the subtypes are distinct from adult rheumatoid arthritis (27).

Several hypotheses can explain the similar phenomenon in myasthenia gravis. The first hypothesis is that they comprise a single disease entity, but the presence of certain HLA antigens precipitates the disease onset, or the presence of HLA antigens are significant only at a certain stage of the immune and neuromuscular system development. It is also possible that the environmental and other acquired pathological factors dilute relative significance of HLA-related factors in the late-onset type. The supporting evidence for this argument is the general similarity of clinical signs and symptoms (marked responsiveness to the Tensilon test, diurnal fluctuation of the symptoms, and characteristic electromyographic findings, etc.) in both childhood and adulthood onset MG.

An alternative explanation is that MG with early childhood onset comprises a completely different subset of the disorder. This hypothesis is more tempting, since the difference in the HLA association in MG compares with that found in diabetes mellitus and rheumatoid arthritis. The other supporting evidence for this hypothesis includes the differences in the fine clinical signs and symptoms between adult-onset and childhood-onset MG $(10,12-16)$. The corticosteroid therapy is more effective and thus complete remission is more common in childhood-onset MG. Mumps infection often leads to complete remission in childhood-onset MG $(12,13)$. However, these clinical differences are rather subtle and still insufficient to establish the childhood-onset MG as a clearly distinct clinical category. To elucidate these contradicting hypotheses, detailed clinical research including comparison of clinical characteristics with HLA types is currently in progress. 
DQA

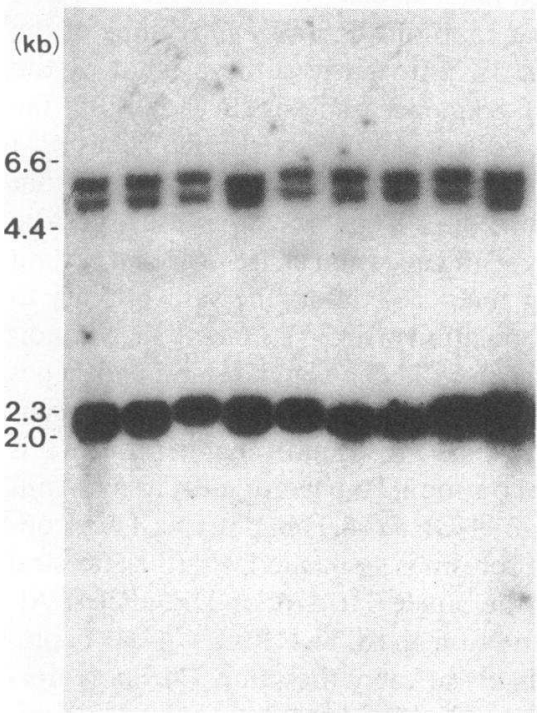

DQB

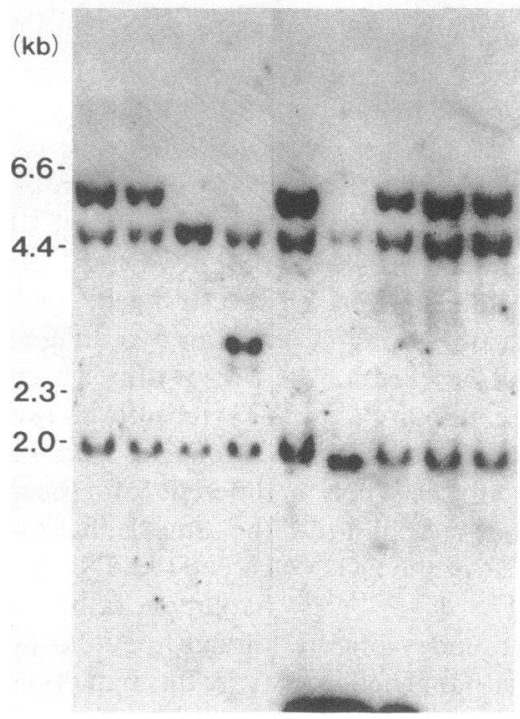

DPB

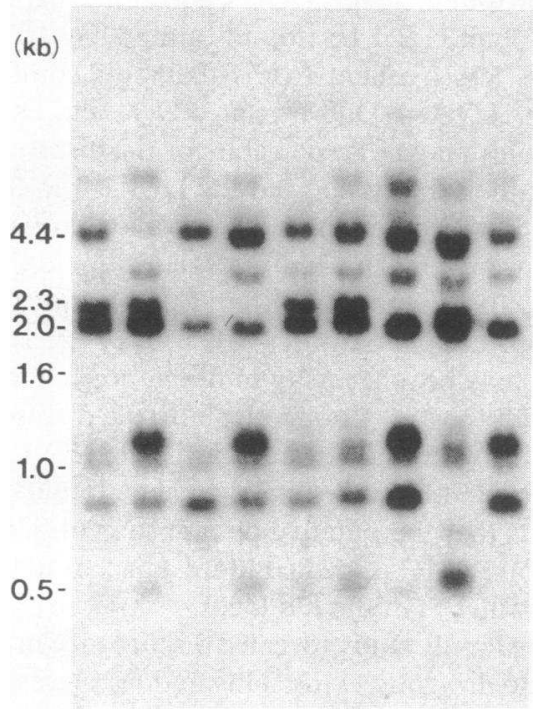

Figure 4. Representative blot of RFLP.

It should be noted that the childhood-onset MG mostly follows the benign course. The early death is rare, most children enter into partial or complete remission, and after a period of successful control, adulthood recurrence is rare (12-16). Thus the "early death" hypothesis that most of the childhood-onset patients die early in their life and that affects the HLA frequencies in adulthood cannot be applied to MG.

In subgroup I, two different statistical analyses showed the presence of synergistic interaction between DR9 and DRw13 (and DQw1 and DQw3). According to the method of Rothman, the relative risk for the DR9/DRw13 heterozygotes was 8.63 times higher than that expected under no synergistic interaction. All the single disease allele models that did not consider synergistic interaction failed to explain the observed HLA frequencies. However, the observed HLA frequencies meshed well with the model proposed by Louis and Thomson that employed synergistic interaction between the multiple disease alleles (26), assuming that DR9 (or DQw3) and DRw13 (or DQw1) act as a dominant and a recessive gene in the absence of the other, respectively.
Although this is the first case of synergistic interaction of HLA antigens reported in Orientals, synergistic interaction is not a very rare phenomenon in the HLA-disease association. In caucasians, increase of heterozygotes of the HLA antigens is observed in insulin-dependent diabetes mellitus $(28,29)$ and celiac disease (30). In a murine model for systemic lupus erythematosus $(31,32)$, heterogeneity of both major histocompatibility complex (MHC) and T cell receptors plays an important role in developing SLE. No previous studies, however, documented the synergistic interaction of HLA alleles in myasthenia gravis.

The mechanism underlying the synergistic interaction is still unknown. One possibility is the formation of unusual $\mathrm{DQ} \alpha / \mathrm{DR} \beta$ or $\mathrm{DQ} \alpha / \mathrm{DQ} \beta$ heterodimers. In celiac disease, the heterodimer composed of the DQ $\alpha$ molecule of the DR3/ DQw2 and DR5/DQw7 haplotypes and the DQ $\beta$ molecule of the DR7/DQw2 haplotype is considered responsible to the disease development (33). In our series, nearly $80 \%$ of the patients examined could have an unusual combination of DQA1 Taq I $6.0 \mathrm{~kb}$ (found in the DQw1/DQw6 haplotypes

Table VII. Number of the Patients with Specific HLA-DR, DQ Combination

\begin{tabular}{|c|c|c|c|c|c|c|c|c|c|c|c|c|}
\hline \multirow{3}{*}{$\begin{array}{l}\text { DQA } \\
\text { Taq I } \\
\text { (kb) }\end{array}$} & \multicolumn{8}{|c|}{ DQB 1} & \multicolumn{4}{|c|}{ DR } \\
\hline & \multicolumn{5}{|c|}{ Eco RI $(k b)$} & \multicolumn{3}{|c|}{ Taq I $(k b)$} & \multicolumn{4}{|c|}{ Serological specificity } \\
\hline & 29 & 20 & 18 & 15 & 2.3 & 6.0 & 3.0 & 1.9 & 9 & 13 & 2 & 8 \\
\hline 6.6 & (4) & 4 & 0 & 0 & 0 & 0 & 0 & 4 & 4 & 0 & (1) & (3) \\
\hline 6.0 & 4 & 17 & 0 & 1 & (14) & (12) & (2) & 18 & 17 & $(12)$ & (3) & 3 \\
\hline 5.4 & 8 & (22) & 1 & 1 & 14 & 13 & 2 & (23) & (22) & 12 & 3 & 6 \\
\hline 4.6 & 0 & 0 & 0 & $(0)$ & 0 & 0 & 0 & 0 & 0 & 0 & 0 & 0 \\
\hline 2.4 & 0 & 1 & (1) & 0 & 0 & (1) & 0 & 1 & 1 & 0 & 0 & 0 \\
\hline
\end{tabular}

All the possible combinations of the DR and DQ genes were examined for 23 patients. Each number in the cell denotes the number of the patients who had the specific combination. Parenthesis denotes the combination usually found in Japanese with strong haplotypic association (24, 31). Among the unusual combinations DQA 1 Taq I $6.0 \mathrm{~kb} / \mathrm{DQB} 1 \mathrm{Taq} I 1.9 \mathrm{~kb}$ was the most frequent. 
[25]) and DQB Taq I $1.9 \mathrm{~kb}$ (found in the DQw9 and other haplotypes [34]). The alternative combination of DQA1 Taq I $5.4 \mathrm{~kb}$ (found in the DQw9 and other haplotypes [25]) and DQB1 Eco RI $2.4 \mathrm{~kb}$ (found in the DQw1/DQw6 haplotypes [33]) is less frequent $(61 \%)$ than this combination. Heterodimers of $\mathrm{DQw} 1 / \mathrm{DQ}$ w6-related $\mathrm{DQ} \alpha$ and $\mathrm{DQw}$ 9-related $\mathrm{DQ} \beta$ molecules may be responsible for the disease development.

All the subjects showed the DQA1 Taq I 5.4-kb and DQB Taq I 1.9-kb fragments. The attributable risks for these fragments were 1.0. This indicates that, should these fragments be absent, there would be little, if any, chance that the subjects develops childhood-onset MG. This suggests that these fragments may be a necessity in developing childhood-onset MG, although they may not contribute the disease-susceptibility as much as other markers such as DR9/DRw13 heterozygosity.

DP (RFLP) was similar to that found in controls. This suggests that the putative disease-susceptibility locus to childhood-onset MG is located more adjacent to the DR or the DQ locus rather than the DP locus.

The family study revealed discordance in two monozygotic and one dizygotic twins. This finding suggests that nongenetic factors are also required in developing MG.

The DR9 haplotypes in the patients were diverse. The second most frequent DR9 haplotype in the patients studied was Cw11-Bw46-BFS-C4A4B2-DR9-DQw3. This haplotype is rare in control Japanese, but found frequently in Chinese MG patients (35). This extended haplotype may play a similar role in developing MG in these two Oriental populations. The diversity of DR9 haplotypes, however, suggests that the specific allele(s) on DR9 haplotypes, such as DR9 and DQw9, would be more important than the haplotype.

The more interesting finding is that nearly $90 \%$ of the DRw13 haplotypes in the patients were C blank-B44-BFFC4A3-C4B1-DRw13-DQw1. Recent studies indicate that this

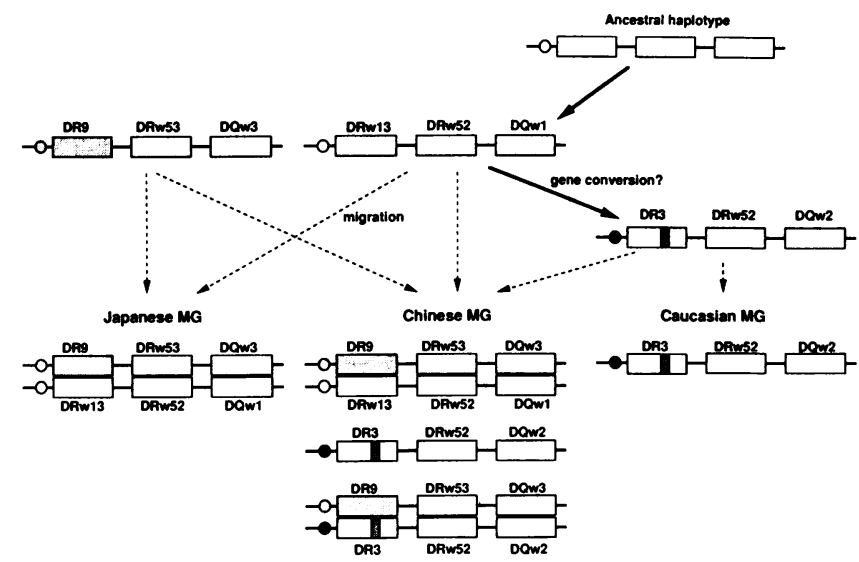

Figure 5. Hypothesis. DR3 and DRw13 are both derived from an ancestral haplotype containing a susceptibility gene to MG (circles). The DR9 haplotype has another susceptibility gene which is different from that in DR3 or DRw 13 haplotype. While DR3-linked susceptibility gene acts as a dominant gene, DR9 and DRw 13 linked genes require each other to develop MG. The DR3 haplotype did not migrate to Japanese and DR9 did not to caucasians, resulting in the different distribution of HLA-linked disease genes and possibly different disease manifestations (especially in the age at onset). It would be interesting to investigate Chinese who have all these haplotypes. However, no extensive study has yet reported for Chinese that includes the patients that developed MG in early childhood. haplotype has a close evolutional relationship with the caucasian B8-DR3-DQw2 haplotype, which is associated with MG in caucasians (5). Pulsed field gel electrophoresis showed that the large-scale molecular map of DR and DQ regions of the B44-DRw13 haplotype is almost the same as that of the B8-DR3 haplotype (36). Sequence analysis suggested that the DR3 DRB gene is derived from the DRw13 DRB genes by a gene conversion event (37). An interesting possibility is that the B44-DRw13-DQw 1 haplotype in Japanese and the B8-DR3-DQw2 haplotype in caucasian share or lack a certain ancestral gene, and that the gene confers the susceptibility to MG in two different populations (Fig. 5). One of such candidates is tumor necrosis factor (TNF). Dawkins and colleagues (38) recently showed that the B8-DR3 (8.1) haplotype in caucasians has a 5.5-kb TNF Nco I restriction fragment, which is different from the more common TNF genotypes $(10.5 \mathrm{~kb})$ but the same as that on the B44-DR4 (44.1) haplotype. They concluded the TNF polymorphism is associated with the ancestral haplotype rather than the single HLA-B or DR alleles. Although they did not study on the B44-DRw13 (44.4) haplotype, this haplotype is likely to carry the same TNF polymorphism, since the B44-DRw13 (44.4) haplotype has more similarity to the B8-DR3 (8.1) haplotype in respect of the large-scale molecular maps than the B44-DR4 (44.1) haplotype does (36). This hypothesis does not necessarily contradict the heterodimer hypothesis, since the effects of this gene could be additive to those of the possible HLA heterodimers.

\section{Acknowledgments}

We are grateful to Ms. M. Okuyama for her excellent technical assistance, and to Dr. P. Nelson for his critical comments.

\section{References}

1. Simpson, J. A. 1981. Myasthenia gravis and myasthenic syndromes. In Disorders of Voluntary Muscle. J. Walton, editor. Churchill Livingstone, London. 585-624.

2. Fritze, D., C. Hermann, Jr., F. Naeim, G. S. Smith, E. Zeller, and R. L. Walford. 1976. The biologic significance of HL-A antigen markers in myasthenia gravis. Ann. N Y Acad. Sci. 274:440-450.

3. Naeim, F., J. C. Keesey, C. Herrmann, J. Lindstrom, E. Zeller, and R. L. Walford. 1979. Association of HLA-B8, DRW3, and antiacetylcholine receptor antibodies in myasthenia gravis. Tissue Antigens. 12:381-386.

4. Pirskanen, R. 1976. Genetic associations between myasthenia gravis and HL-A system. J. Neurol. Neurosurg. Psychiatry. 39:23-33.

5. Tiwari, J. L., and P. I. Terasaki. 1985. HLA and Disease Association. Springer-Verlag, New York. 472 pp.

6. Dawkins, R. 1981. Myasthenia gravis. In Histocompatibility Testing 1980. P. I. Terasaki, editor. UCLA Tissue Typing Laboratory, Los Angeles, CA. 662-667.

7. Yoshida, T., M. Tsuchiya, A. Ono, H. Yoshimatsu, E. Satoyoshi, and K. Tsuji. 1977. HLA antigens and myasthenia gravis in Japan. $J$. Neurol. Sci. 32:195-201.

8. Ono, A., K. Kurita, M. Tsuchiya, I. Yamanaka, and K. Tsuji. 1975. HLA antigens of intrathymic and peripheral lymphocytes in autoimmune disease. Keio J. Med. 24:367-376.

9. Hirayama, H., and T. Sugishita. 1974. Epidemiological study of myasthenia gravis of Japan. In Proceedings of Research Committee on Etiology, Treatment and Prevention of Myasthenia Gravis, the Ministry of Health and Welfare of Japan, Tokyo. 50-66.

10. Fukuyama, Y., Y. Hirayama, and M. Osawa. 1981. Epidemiological and clinical features of childhood myasthenia gravis in Japan. 
In Myasthenia Gravis. Pathogenesis and Treatment. Japan Medical Research Foundation, editor. University of Tokyo Press, Tokyo. 19-27.

11. Igata, A. 1987. Diagnostic criteria for myasthenia gravis. In Proceedings of Neuroimmunological Disorders Research Committee 1986. A. Igata, editor, the Ministry of Health and Welfare of Japan, Tokyo. 1-4.

12. Segawa, M. 1986. Treatment of myasthenia gravis. Chiryo. 68:1359-1366.

13. Segawa, M. 1986. Treatment of myasthenia gravis-discussion on the age at onset. J. Therap. 68:1359-1366.

14. Segawa, M., Y. Nomura, and E. Nagata. 1985. Myasthenia gravis (MG) in childhood-age at onset and pathogenesis. J. Neurol. 232(Suppl):59. (Abstr.)

15. Segawa, M., Y. Nomura, M. Soda, A. Uchiyama, K. Matsuki, and T. Juji. 1990. Long-term prognosis of myasthenia gravis of childhood-its relation to age at onset and HLA typing. In Neuroimmunological Diseases-Recent Advances in Pathogenesis and Treatment. Tokyo University Press, Tokyo. 373-376.

16. Nomura, Y., M. Segawa, M. Soda, A. Uchiyama, K. Matsuki, T. Juji, and N. Shimoyama. 1990. HLA typing and T-cell subsets in myasthenia gravis of childhood. In Neuroimmunological DiseasesRecent Advances in Pathogenesis and Treatment. Tokyo University Press, Tokyo. 89-92.

17. Tokunaga, K., K. Omoto, Y. Fujii, and T. Jiji. 1983. Haplotype analysis on HLA-linked genetic markers. Ishoku. 18:179-189.

18. Matsuki, K., T. Juji, K. Tokunaga, T. Naohara, M. Satake, and Y. Honda. 1985. HLA haplotype frequencies estimated from the data on HLA class I, II, and III antigens in 111 Japanese narcoleptics. $J$. Clin. Invest. 76:2078-2083.

19. Sim, E., and S. J. Cross. 1986. Phenotyping of human complement component C4, a class-III HLA antigen. Biochem. J. 239:763767.

20. Alper, C. A., T. Boenisch, and L. Watson. 1972. Genetic polymorphism in human glycine-rich beta-glycoprotein. J. Exp. Med. 135:68-80.

21. Wolf, E., K. M. Spencer, and A. G. Cudworth. 1983. The genetic susceptibility to type 1 (insulin-dependent) diabetes. Diabetologic. 24:224-230.

22. Rothman, K. J. 1976. The estimation of synergy or antagonism. Am. J. Epidemiol. 103:506-511.

23. Thomson, G. 1983. Investigation of the mode of inheritance of the HLA associated diseases by the method of antigen genotype frequencies among the diseased individuals. Tissue Antigens. 21:81-104.
24. Thomson, G. 1981. A review of theoretical aspects of HLA and disease association. Theoretical Population Biol. 20:168-206.

25. Matsuki, K., H. Maeda, T. Juji, H. Inoko, A. Ando, K. Tsuji, and Y. Honda. 1988. Taq I-generated HLA-DQ $\alpha$ polymorphism in Japanese patients with narcolepsy. Immunogenetics. 27:87-90.

26. Louis, E. J., and G. Thomson. 1986. Three-allele synergistic mixed model for insulin dependent diabetes mellitus. Diabetes. 35:958-963.

27. Brewer, E. J., E. H. Giannini, and D. A. Person. 1982. Juvenile Rheumatoid Arthritis. W. B. Saunders Co., Philadelphia. 351 pp.

28. Nerup, J. 1978. HLA studies in diabetes mellitus: A review. Adv. Metab. Disord. 9:263-277.

29. Woolf, B. 1955. On estimating the relation between blood group and disease. Ann. Hum. Genet. 19:251-253.

30. Tiwari, J. L., H. Betuel, L. Gebuhrer, and N. E. Morton. 1984. Genetic epidemiology of coeliac disease. Genetic Epidemiol. 1:37-42.

31. Yanagi, Y., S. Hirose, R. Nagasawa, T. Shirai, T. W. Mak, and T. Tada. 1986. Does the deletion within $T$ cell receptor $b$-chain gene of NZW mice contribute to autoimmunity in (NZB $\times$ NZW) F1 mice? Eur. J. Immunol. 16:1179-1182.

32. Hirose, S., G. Ueda, K. Noguchi, T. Okada, I. Sekigawa, H. Sato, and T. Shirai. 1986. Requirement of H-2 heterozygosity for autoimmunity in (NZB X NZW)F1 hybrid mice. Eur. J. Immunol. 16:1631-1633.

33. Bugawan, T. L., G. Angelini, J. Larrick, S. Auricchio, G. B. Ferrara, and H. A. Erlich. 1989. A combination of a particular HLADP $\beta$ allele and an HLA-DQ heterodimer confers susceptibility to coeliac disease. Nature (Lond.). 339:470-473.

34. Numaga, J., K. Matsuki, M. Mochizuki, M. Minami, and T. Juji. 1988. An HLA-D region restriction fragment associated with refractory Behçet's disease. Am. J. Ophthal. 105:528-533.

35. Hawkins, B. R., C. L. Wy, C. Ekk, and H. Ay. 1984. Strong association of HLA Bw46 with juvenile onset myasthenia gravis in Hong Kong Chinese. J. Neurol. Neurosurg. Psychiatry. 47:555-557.

36. Tokunaga, K., P. H. Kay, F. T. Christiansen, T. Saueracker, and R. L. Dawkins. 1989. Comparative mapping of the human major histocompatibility complex in different racial groups by pulsed field gel electrophoresis. Hum. Immunol. 26:99-106.

37. Rollini, P., B. Mach, and J. Gorski. 1985. Linkage map of three HLA-DRb-chain genes: Evidence for a rare duplication event. Proc. Natl. Acad. Sci. USA. 82:7197-7201.

38. Dawkins, R. L., A. Leaver, P. U. Cameron, E. Martin, P. H. Kay, and F. T. Christiansen. 1989. Some disease associated ancestral haplotypes carry a polymorphism of TNF. Hum. Immunol. 26:91-97. 